\title{
Connecting science education to a world in crisis
}

Kenneth Tobin

Correspondence: ktobin0@gmail.com The Graduate Center, City University of New York, 365 Fifth Avenue, New York, NY 10016-4309, USA

\section{Springer}

\begin{abstract}
Even though humanity faces grand challenges, including climate change, sustainability of the planet and its resources, and well-being of humans and other species, for the past 60 years science educators have been preoccupied with much the same priorities. Adherence to the tenets of crypto-positivism creates problems for research in the social sciences (e.g., over reliance on statistical analyses leads to oversimplified models that strip away context and are reductive). Hypotheses and associated statistical tests support causal models that rarely predict social conduct or blaze pathways for meaningful transformation. In contrast to the mainstream of research in science education, I advocate a multilogical methodology that embraces incommensurability, polysemia, subjectivity, and polyphonia as a means of preserving the integrity and potential of knowledge systems to generate and maintain disparate perspectives, outcomes, and implications for practice. In such a multilogical model, power discourses such as Western medicine carry no greater weight than complementary knowledge systems that may have been marginalized in a social world in which monosemia is dominant. I describe research methodologies that have the potential to transform science education and our ongoing research in urban science education. I show how our research evolved to include studies of science for literate citizenry - expanding foci to address birth through death and all settings in which learning occurs - not just schools. Our research aims to be transformative since it includes interventions developed to use what we learned from research to ameliorate intense emotions, improve learning, and enhance the well-being of participants. I explain how we incorporated Jin Shin Jyutsu, a complementary medical knowledge system, to ameliorate intense emotions, become mindful, and improve well-being of participants. I also address research on meditation and mindfulness and their potential to improve learning, emotional styles, and wellness. In a final section I address three of the most important questions raised by colleagues, including scholars from Asia, as I exhort science educators to address grand challenges that threaten the Earth and its social institutions - the alternatives are catastrophic.

Keywords: Emotions, Mindfulness, Meditation, Cogenerative dialogue, Multilogicality, Authentic inquiry, Event-oriented inquiry, Jin Shin Jyutsu
\end{abstract}

\section{Connecting science education to a world in crisis}

Nothing is permanent - not even science education! This thought has been with me for a while as the relentless cycle of reform/transform continues to beat its drum as it reproduces sameness with little change (Hurd 1997). With this thought in mind I clicked on the BBC icon to check up on the news. Many items caught my attention, most of them relevant to this paper. Yet another study was warning of the threat of vertebrate extinctions, including

(c) 2015 Tobin. Open Access This article is distributed under the terms of the Creative Commons Attribution 4.0 International License (http://creativecommons.org/licenses/by/4.0/), which permits unrestricted use, distribution, and reproduction in any medium, provided you give appropriate credit to the original author(s) and the source, provide a link to the Creative Commons license, and indicate if changes were made. 
humans. The Earth has entered a new phase of mass extinctions according to a report that vertebrates are disappearing more than 100 times faster than normal. Unlike the last mass extinction, 65 million years ago, when damage was likely attributed to a collision with a large meteor, on this occasion the problems are connected to climate change, pollution, and deforestation. In the paper that was the focus of the BBC report, Gerardo Ceballos, Paul Ehrlich, Anthony Barnosky, Andrés García, Robert Pringle, and Todd Palmer (Ceballos et al. 2015) remarked:

Avoiding a true sixth mass extinction will require rapid, greatly intensified efforts to conserve already threatened species and to alleviate pressures on their populations -notably habitat loss, overexploitation for economic gain, and climate change. All of these are related to human population size and growth, which increases consumption (especially among the rich), and economic inequity. However, the window of opportunity is rapidly closing.

I begin this paper in this way because of the note about the rapidly closing window of opportunity. This is an alarm bell for science educators and policy makers. Sustainability is an ideal context because it is an overarching issue for wellness, climate change, and mass extinctions. These are some of the grand challenges that humanity faces and education is clearly central to success (Powietrzynska et al. 2015). Oddly enough, despite reports and associated research, the issues have not gained traction among science educators and most policy makers. Rhetoric does not translate into science education - for everyone, all the time, everywhere. For example, I have not felt the pressure to educate the public to mobilize the actions necessary to avert a sixth mass extinction. Furthermore, there seem to be low levels of awareness that humans are among the vertebrates that could become extinct. The political leadership in the US seems confined to rhetoric, and in Australia (a country of which I am a citizen), there is still a high level of denial about issues like climate change and responsibilities of humans to address sustainability. Scientism, crypto-positivism, neoliberalism, and meritocracy, among other factors, support an ideology, a common sense, that argues for reform but achieves more of the same (Kincheloe and Tobin 2009).

The alarm bells are ringing loudly, but will they be heard? As John Donne ${ }^{1}$ asked in 1624, "Perchance he for whom this bell tolls may be so ill, as that he knows not it tolls for him?" (1624, p. 108). Will the science education community take heed of the alarm bells and mobilize new forms of action? It seems as if those who are alert are already acting decisively (Mueller and Tippins 2015) - but they are a small minority. The mainstream seems preoccupied with business as usual. For whom does the bell toll? Science educators should ask, for I fear it tolls for them.

\section{How does our research relate to the grand challenges?}

Since I began a program of research in science education in 1973 I have endeavored to address socially significant issues. Initially our research focused on teaching, teacher education, and learning. For more than four decades we changed theoretical frameworks and methodologies to ensure that our research was relevant to local, national, and international stakeholders. As we did so, our research foci changed in an endeavor to keep up with rapidly changing social priorities. Throughout this period, a concern was that many of my colleagues continued to study much the same issues over their entire career. I do not doubt 
their credibility as scholars. The methodologies they adopted were saturated with crypto-positivism and reductive methods greatly expanded the questions arising out of each completed study. They were caught in an inward spiraling process they felt would illuminate social truths that would eventually improve the quality of science education. My concern alerted me to a necessity to always ask of my own scholarship - is this research worth doing?

In the past 15 years we adopted a methodology of authentic inquiry. The approach is collaborative and seeks to involve all participants as co-researchers. Authentic inquiry aims to address the goals of all stakeholders, not just a privileged few. Importantly, theory and practice are considered to be dialectically related constituents of a whole, neither privileged over the other, with all participants expected to benefit from the research in which they are involved. An emergent and contingent design embraces respect for difference while seeking to understand what is happening, why it is happening, and how changes might be made to afford positive, collective and individual changes.

Since our research incorporated a multilogical methodology built upon a sociocultural foundation it was apparent that what was happening in classrooms, pre-K through college, was a microcosm for lifestyles in society writ large. Emotions, produced in the moments of enacting everyday practices, more often than not, attached themselves to conduct, became stuck, and mediated what happened and transformed social fabric in ways that often were deleterious. It also was apparent that emotions often built to excesses that negatively impacted wellness. That is, the focus we had established on expressed emotions and wellbeing of teachers and students was seen in a larger social context as a central tenet of literate citizenry - applicable to all humanity on a life through death continuum. We related our research to sustainability, focused on human wellness with the aim of catalyzing lifestyle changes to create and maintain harmony while enacting social life to maximize well-being of selves and others. The grand challenge that provides an umbrella for our ongoing research is to educate the public to reduce the threat of mass extinctions.

\section{Overview}

In this paper I trace pathways of our research projects in science education, since about 1998, in a context of research on emotion and its evolution into studies of wellness and especially the links between emotions and wellness. I then address methodologies we have employed to support research that is increasingly sociocultural in its foundations and collaborative with research participants. Finally, I look ahead in a context of studies on wellness and the desirability to examine what is happening and why it is happening in terms of complementary knowledge systems that can provide expansive possibilities for viewing and resolving issues of wellness. My use of Jin Shin Jyutsu, a knowledge system whose roots are several thousand years old, draws attention to complementarity as a guiding tenet rather than what is true or what is best. Jin Shin Jyutsu provides a fresh way to look at emotion and wellness and offers possible interventions that people can use to reduce excess emotions, resolve personal health issues, and aid others.

\section{Multilogicality}

Illuminating social life

Theory can be considered as a light on social reality - illuminating what is happening and providing a rationale for understanding why it is happening (Tobin 2008). Since 
theory can address such questions it is a tool for interpretive researchers to seek to understand social life, from the perspectives of participants. The role of an interpretive researcher is to find out from participants what is happening and the rationale for what is happening. A theoretical framework illuminates social reality, but it only shows what the theory is capable of showing. Different theories can provide different landscapes for what is happening and why it is happening. For example, theories about expressed emotions might give interesting accounts of facial expression of emotion, emotions represented by prosody, and use of gestures when emotions become excessive. However, as rich as these insights might prove to be they will be silent about more issues than they illuminate. As theories illuminate so they obscure. How do people act to restore harmony in their bodies when their blood oxygen is low, when pulse rate is high, or when body temperature rises? Narratives about mindfulness might be absent because participants are not aware that mindfulness is salient to what is happening - or they might not even know there is such a thing as mindfulness. Becoming aware of a particular theory that might have salience to what is happening and why it is happening affords seeing differently. Of course, if participants see differently there is a fresh stream of possibilities for action - depending on what they see and their value system in relation to what they see happening, they will act accordingly. That is, fresh ways of looking can expand action possibilities. Becoming aware of what participants previously were unaware of is a way of fracturing existing equilibria - akin to an intervention that can unsettle patterns of action in particular social settings. Ann Swidler (1986) noted that culture reveals itself in unsettled times - an insight that provides a rationale for interventions that expand theoretical repertoires, thereby enabling participants to reflexively engage with their lifeworlds - becoming aware of things they previously were unaware of, and making changes when and as they feel change is necessary.

Expanding the role of interpretive research to include reflexive intervention is consistent with Pierre Bourdieu's reflexive sociology (1992) and Harold Garfinkel's ethnomethodology (1967). Also, it is aligned with authentic inquiry; an approach to research that expands opportunities for participants and their associated institutions to benefit from being involved in research. In this particular instance reflexive practices can result in participants changing their ontologies. This allows them to see what others know and do in a different light, opening doors for them to learn new things from and about others. These processes of learning about self and others through reflexive hermeneutic phenomenology transform and can improve social life for selves, others, and various collectives with which participants are associated.

\section{Event oriented inquiry}

William Sewell Jr.'s paper on agency and structure (1992) almost escaped my attention when, as a senior scholar, I was studying theoretical sociology courses at the University of Pennsylvania. A doctoral student from the School of Education asked me if I had read Sewell's article, and when I said I had not, he encouraged me to take a look at it. The paper was one of many that had been assigned by a professor as reading for one of the 15 weeks of a doctoral course on social theory. In any given week there were far more assigned readings than I could address. Accordingly, if the student had not drawn my attention to the paper, I very much doubt I would have read it. 
The student was right on target. Sewell addresses agency $\mid$ structure $^{2}$ relationships and led several colleagues and me to read the paper and use it as part of a multilogical framework for our research. We searched articles Sewell had published and his theories of culture became central to our work (e.g., 1999). Importantly, Sewell (1997) compared his own position on theories of culture to the work of Clifford Geertz (1973) and drew our attention to the dialectical relationships between patterns and contradictions as well as schemas and practices. Since Frederick Erickson (1986) had embraced Geertz' theories of culture in his work on interpretive research, it was necessary for us to rethink interpretive research as methodology and address implications of culture being characterized by patterns having thin coherence and associated contradictions.

From a Geertzian perspective contradictions were regarded as exceptions - to an assertion for which there was overwhelming support (i.e., forming a pattern that had thick coherence). Accordingly, the challenge for an interpretive researcher was to explain the exception to the assertion - almost as if the assertion were a social truth. In so doing the narrative in which assertions and contradictions were embedded were nuanced and more complex than was the case in statistical research, where deviations from a grand mean were often considered to be errors. In an important way the Geertzian model pointed toward monosemia - that is, a social reality in which there was a truth to be identified, hopefully through empirical research. In contrast, our research program focused on the challenge of understanding difference in terms of polysemia - whereby individuals who were placed differently in social space experienced different social realities. Accordingly, individuals provided evidence in research that reflected their lived experiences, and empirical research produced differences that reflected different social realities (i.e., social truths). Our adoption of this way of thinking about difference was clearly at odds with the perspectives on culture articulated by Geertz.

In 2005, Sewell published a book containing his key works (2005). In this volume we identified a fourth contribution from Sewell that was to become central for our research. This chapter introduced event-oriented social theory. Essentially an event was considered to be transformative and salient to the participants. Since we considered all cultural production as both transformative and reproductive we focused on the most important characteristic of event to be its salience to participants. A metaphor to provide insights into the meaning of event is a "spike in the curve." Events, or spikes in the curve, occur within episodes, which are within a lesson. Once identified, a spike can be contextualized in relation to the episode in which it is situated/contained. Over a relatively short period of time event-oriented inquiry became a core component of our multilogical research methodology (Tobin and Ritchie 2011). It is to be noted that identifying events is a multilevel exercise that can be focused on micro, meso, and macro levels. Most of our research involves multilevel analysis, using participatory methods and resources such as digital video and audio data, and finger pulse oximeters. At the meso and macro levels we employ cogenerative dialogue, video/ audio recording, participant observation, and a variety of heuristics, among other data resources.

\section{Authentic inquiry}

For the past decade I have collaborated with colleagues to develop a research methodology that is multilogical, built on a foundation that accepts as primary the tenet that ontologies derive from lived experiences, and as such, they are socially constituted. Ontologies reflect 
lived experiences, hence histories, including labels that place individuals in an ndimensional social space (e.g., race, ethnicity, gender, sexual orientation, age, native language, and place of residence). As a researcher, I can use theories as tools to illuminate social life in ways that accord with my axiological commitments ${ }^{3}$. However, since we accept poly-ontology (i.e., the co-occurrence of multiple realities/many ways to answer the question: What happened?), we acknowledge that the frameworks we use, with and without awareness, illuminate and obscure what we experience as researchers. That is, our research frameworks are subjectivities that orientate and inform, but are not unique and should not be privileged to justify inequalities and social harms.

An overarching framework for our research is authentic inquiry. Our journey toward fully utilizing this framework began in the 1970s when we first learned about genetic epistemology and its applicability to designing and enacting science curricula. Our reading of Jean Piaget began a journey into learning and teaching that orientated toward constructivism, understood in the context of learning and teaching science in ways that acknowledged an individual's learning as socially mediated (1964). The journey was characterized by several steps along the way in which it was necessary to resolve issues such as stages of reasoning, conceptual change, and social knowledge. Inevitably there were distractions and sidebar debates (e.g., especially with critics of radical constructivism, including Michael Matthews and Denis Phillips) that undoubtedly expanded our understandings of research on teaching and learning (e.g., Matthews 1998; Phillips 1983).

In the 1990s we first learned about the authenticity criteria, as Egon Guba and Yvonna Lincoln (1989) proposed them, as a methodology for evaluation. These four criteria (i.e., ontological, educative, catalytic, and tactical authenticity) became foundational for what we would later describe as authentic inquiry, which is a complex, multilogical framework that embraces ethical conduct characterized by care, compassion, honesty, courage, social justice, and autonomy. A multilectical stance considers all social constructs associated with authentic inquiry as constituents of a whole, each presupposing the existence of the other (Fellner 2014). The meanings of these categories arise from our uses of them for almost two decades, including informative dialogues with Joe Kincheloe about authentic inquiry as multilogicality and non-acceptance of Thomas Kuhn's incommensurability (1996). In the following sections I describe four categories, emphasizing that any attempt to describe one authenticity criterion inevitably cycles through descriptions of the others.

\section{Ontological authenticity}

Ontological authenticity emphasizes the necessity that all participants change their ontologies in the process of doing research. Accordingly, we adopt a form of research that is emergent and contingent. That is, we study salient events and learn from them. In so doing we are alert to the necessity to always nuance claims we would make that might be interpreted causally. We are conscious that what we learn will necessarily be incomplete because of a crisis of representation which points to the transcendence of knowledge - which cannot be fully represented. Also, we adopt a theoretical stance that all claims based on patterns of coherence (as interpreted from data analysis), are accompanied by contradictions. The patterns and contradictions coexist (i.e., they presuppose one another) and are part of the learning that should occur in a study (i.e., learning considered as cultural production) (see Tobin 2015 for a fuller description of authentic inquiry). 
All stakeholder groups should learn from ongoing research, not just officially designated researchers. Accordingly, provision should be made in the research design to show how participants' ontologies within different stakeholder groups change progressively throughout a study. In this way research that involves authentic inquiry will be transformative since all participants change their ontologies, as documented in the research. Consistent with an acceptance of difference, ontological shifts would be represented with many voices and research design would embrace polysemia (i.e., many meaning systems, hence truths).

\section{Educative authenticity}

Regular seminars can be scheduled to allow research participants to educate one another. The purpose is to educate all participants about standpoints held by others - not to persuade them to change to a particular (i.e., different) set of understandings. A characteristic of authenticity embraces difference and values opportunities to learn from others' different perspectives. The educative criterion specifies that each stakeholder group should learn about and from others' ontologies. That is, the design of the study should provide opportunities for all stakeholder groups to learn from one another. We have found cogenerative dialogue to be a particularly useful activity to afford educative authenticity.

As stakeholders participate in social life, what they learned from research can be used by them, with and without conscious awareness, and can educate those with whom they interact. We refer to this process as ripple effects - a way to disseminate what is learned from research to participants in other fields in which research participants are involved. Central to this idea is the notion that fields do not have boundaries and structures emanating from any field of the lifeworld can mediate what happens in other fields of the lifeworld. In this way research can be regarded as transformative in a multilevel sense (i.e., macroscopically).

\section{Catalytic authenticity}

A conscious goal, beyond changing ontologies and understanding one another's ontologies is to take what is learned from participation in research to catalyze improvements in the institutions involved in the research. The design of the research should be such that adjustments can be made as the research progresses, to ensure that opportunities to transform and improve are planned and enacted. In this way research design is contingent and emergent, always being attentive to ongoing interpretations and diverse perspectives that can make visible possibilities for institutional advancement. The design and use of interventions became an integral part of our ongoing research.

\section{Tactical authenticity}

The goal of catalytic authenticity also can be pursued at the level of individuals. That is, individuals should take what is learned from research to transform their practices and benefit accordingly. However, some individuals are not well placed to access and appropriate what is learned from research. Accordingly, those involved in the research, who are well positioned to improve equity, should act to help those who are not appropriately placed to take advantage of what is being learned from the research. There are many scenarios that might apply, but most importantly, those with the power to transform should do so, using what is learned from research to improve the social lives of those who would potentially benefit from the changes. This goal avoids a situation in which researchers are 
learning but do not make efforts to improve social justice, based on what they have learned from the research. Authentic inquiry embraces an ethical responsibility to act to benefit others, especially those who are not ideally placed to benefit from what has been learned from research. At the heart of tactical authenticity is reflexivity, becoming aware of the unaware, watching out for recurrences of patterns of disadvantage, and acting with conviction to the benefit of those who are disadvantaged (Bourdieu 1992). In a context of wellness we carefully monitored blood oxygenation levels and pulse rate of coteachers and, as they approached thresholds of concern, we intervened so that teachers would not risk initiating health crises. Most commonly, this involved surprisingly low levels of blood oxygenation (i.e., less that $90 \%$ and in some cases less than $70 \%$ ).

The adoption of authentic inquiry as core methodology drew attention to a multilectical orientation in which all four components are co-present constituents of a whole. That is, ontological and educative authenticity with their orientations toward changes in theories held by research participants are co-present with catalytic and tactical authenticity that orientate toward changed practices. Research with an authentic inquiry orientation values and incorporates changes in theory and practices as co-present constituents of a whole. The perspective embraces an individual | collective relationship.

It is important to note too, that a strength of authentic inquiry is that it assumes that individuals, as social entities, are different and will remain so in ways that reflect their held epistemologies, ontologies, and axiologies. Such differences are not regarded as inconveniences but instead are embraced as resources for learning in a nuanced way. The value for learning from difference has implications for other inclusions in the methodological bricolage we employ in our research. Also central is dialogue as a characteristic of verbal interaction within and between individuals. That is, speech, whether it is inner or outer, has a recursive nature that is expansive in the sense that it opens up fresh ways of thinking about what is known, what is happening, what is valued, and what is possible and desirable (Bakhtin 1986). Accordingly, research methodology also includes the activity of cogen, which incorporates dialogue, collaborating with others, radical listening, right speech, mindfully speaking, and learning from difference.

\section{Cogenerative dialogue}

My research on speaking and listening productively started in 1973 with a study on the ways in which silence between utterances, specifically teacher wait time, could enhance science achievement and quality of teacher and student participation in science (Tobin and Capie 1982) and mathematics (Tobin 1986) classes. Years later, in a collaborative two-country study on coteaching, with Wolff-Michael Roth, we developed a collaborative activity which we called cogenerative dialogue (hereafter cogen). The number of participants in cogen can vary from two upward and selection usually seeks to maximize different perspectives (Tobin and Roth 2006). Representatives from different stakeholder groups involved in a science lesson would meet to discuss what happened, why it happened, and what needs to be done to enhance the quality of the learning environments and achievement. Although we focused in the initial stages of this research on reaching consensus on what had to be done in the next lesson, we also created rules that would define cogen. Since the developmental days in the late 1990s, the characteristics of cogen have been adapted, and some of the most salient are listed in Fig. 1 (Tobin and Alexakos 2013). The research using cogen has been ongoing and the purposes of cogen have 


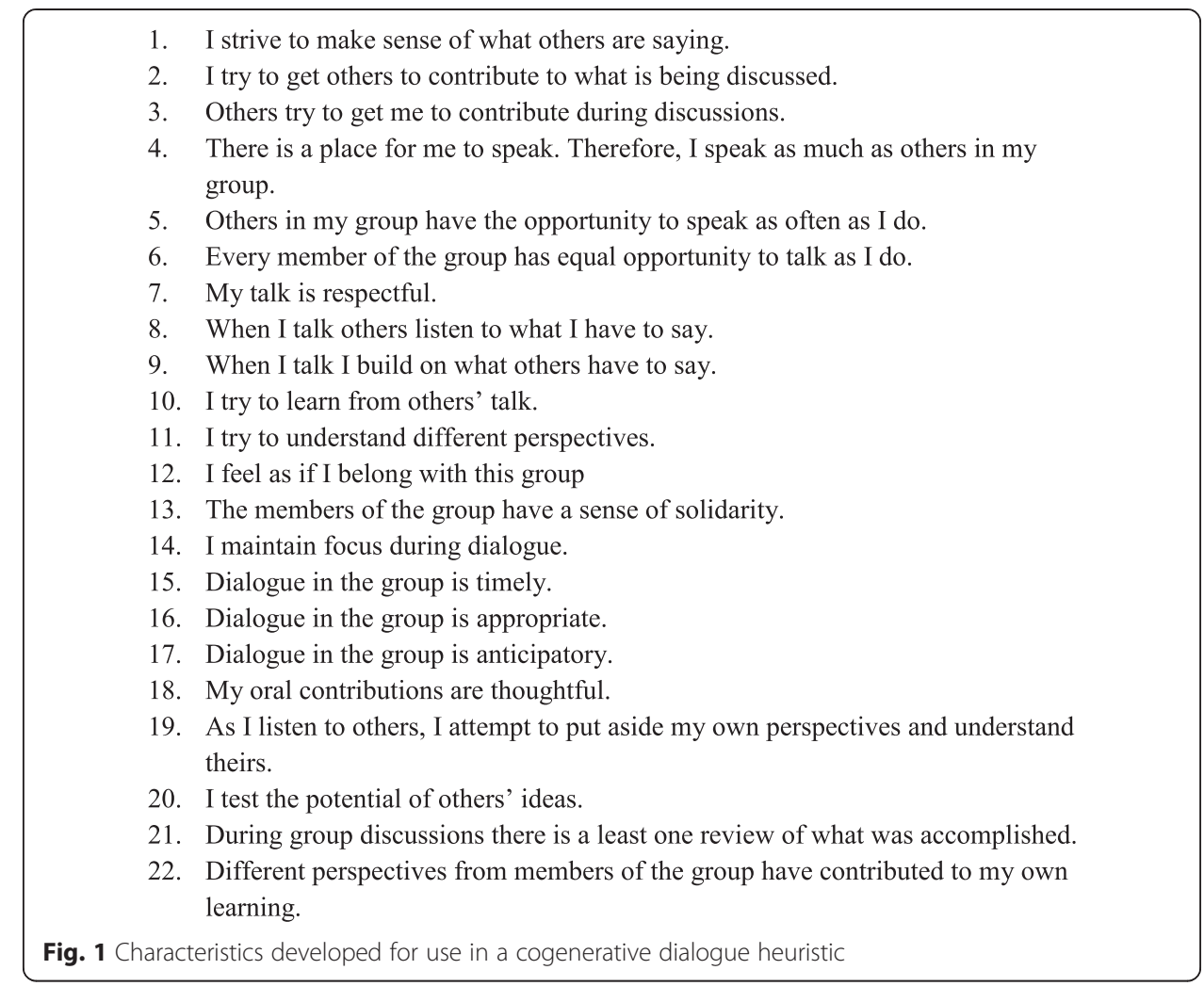

been expanded to take advantage of it being a field for the production (i.e., transformation | reproduction) of adaptive forms of culture that afford success in fields characterized by important social differences. In the 15 years of the current millennium the ongoing research has examined cogen from pre-K through graduate school and in settings outside of schools. Also, cogen has been consistently and successfully used as a research methodology in which all stakeholders can engage in research.

\section{Generalizability}

Our preference was to broaden the understanding we held about the roles of researchers and participants. To the extent possible we want all research participants to identify as researchers and enact roles within a framework of authentic inquiry. The orientation of the research was toward events that emerged in everyday life. For example, in a science classroom, we endeavor to involve participants in personal and collective inquiry that is an integral part of ongoing research. By expanding participants' roles the likelihood of the study being authentic is greatly increased.

We regard researchers as learners, rather than truth seekers. In the process of learning care is taken to nuance claims in ways that identify and respect many forms of difference. A framework that all participants use while doing research is a bricolage consisting of authentic inquiry, emergence and contingence, post structural hermeneutics, and event oriented inquiry.

As participants learned from the research they use cogen to explore ways in which what they have learned can improve the quality of social life, not only for themselves, but also 
for others with whom they interact. Enacting what has been learned from research, as appropriate, throughout the lifeworld is regarded as a central part of authentic inquiry, especially in relation to catalytic and tactical authenticity criteria. Participants enact what they have learned to transform the fields of their lifeworlds and create possibilities for others to learn from them by being with them (at the elbows). Thus, enactment in other fields is a form of dissemination of schemas and practices that have emerged as "learning from research." Since others were involved agentically and passively, by being-in-with research participants, the activity serves as an example of the generalizability of authentic inquiry, and also its applicability to lifeworlds - not just to the specific fields that were targeted as the initial sites for the research. In this way the research is expansive and relevant to everyday life. Importantly, the ripple effects of the research into participants' lifeworlds raise possibilities for enhancing literate citizenry. Furthermore, the involvement of many others further expands the design of the research and its continuing possibilities.

\section{Research on emotions}

Research on emotions became central to our research when I assumed a position in an urban education program at the University of Pennsylvania, in 1997. Partly due to problems my teacher education students experienced when they taught science in inner-city schools in Philadelphia, I initiated a program of research in urban high schools. The purposes of the research embraced authentic inquiry. That is, my presence as a teacher researcher was intended to educate me about teaching and learning science in West Philadelphia while, simultaneously, high school youth would learn science, their regular teacher would learn more about teaching, learning and research, and my students and colleagues at the University of Pennsylvania and elsewhere would learn from our research and teaching (Tobin 2000). The study involved numerous faculty and graduate students and continued in Philadelphia for six years and still is ongoing in New York and New Jersey (Tobin et al. 2001).

The initial framing of research on emotions was grounded in the work of Randall Collins (2004) and Jonathan Turner (2002). Our interests focused on the incidence of positively and negatively valenced emotions and ways in which they were transmitted through the classroom. In our studies of the expression and transmission of emotions we explored rituals and ways that emotions appeared to saturate social artifacts to afford reproduction when similar structures unfolded in the future. Accordingly, we applied methodologies and associated methods to expand our research to follow emerging (i.e., continuously changing) pathways that were contingent on who was doing the research, the use of collaborative models, and the specifics of the multilogical frameworks we employed. As the research unfolded our foci included emotional climate, prosody, and proxemics (Bellocchi et al. 2014).

\section{Expression of emotion}

The uses of digital video expanded the possibilities for microanalysis of video and also audio files. Following the work of Collins (2004) we began studies of the ways in which synchrony and entrainment occurred during verbal interaction in different types of activity. Studies of prosody were interrelated with our interests in emotional expression. Accordingly, we examined patterns in utterances, including energy distribution as a function of time (Roth and Tobin 2010). There is considerable work still to be done as 
far as prosodic expression of emotion is concerned. We are interested in the energy distribution in different formants (i.e., harmonics) as emotions change in type and intensity. Also, as others detect variations in prosody we anticipate that through social resonance they will produce similar emotions to those expressed by the speaker. Our goal is to better understand emotional contagion through the lenses of social resonance and empirical analyses of the dispersion of energy in the voice as emotions vary in nature and intensity.

We need to know more about the expression of emotion through different modalities. It seems likely that emotions can be suppressed through a person's agency. For example, someone who is very excited might suppress facial expressions, but not pulse rate, body temperature, and blood oxygenation. Similarly, anger in the voice might be masked by carefully measuring each word, the face may present neutral emotions, but disharmonies might occur in the body due to stress and palpitations. The domain of physiological expression of emotions is a fertile area for research.

Since we have been learning about Jin Shin Jyutsu (JSJ) we have been analyzing video of social settings to see if participants control emotional intensity and expression through the use of JSJ-like touches and holds (Tobin 2015). There is ample evidence of JSJ-like touches and holds being used in everyday life and the next steps in the research are to ascertain why these touches and holds are used and what they accomplish (Tobin et al. 2015).

\section{Teaching makes me sick}

Our research in the Bronx of New York City was in many ways reminiscent of our research in West Philadelphia - in which I had been a teacher researcher. We learned a great deal in both studies, especially about the role of expressed emotions in relation to the quality of learning environments. What seems different in the study in the Bronx research is that the primary teacher in our work frequently displayed intense anger even in cogens - which were supposed to be dialogic (Tobin and Llena 2011). We often talked about the intensity of the teacher's anger and his insistence that he was acting that the anger was not real. However, we maintained that it seemed the teacher's body might not have known the difference between acting out anger and actually being angry. His stress levels seemed to be elevated and we were concerned that his wellness suffered. Inevitably it seemed, he had heart problems and eventually had to resign from teaching based on medical advice that continuing to teach could prove to be fatal.

\section{Dissolving boundaries}

Research on emotions heightened our awareness of the connection between emotions and health. Accordingly, it became a priority for us to develop interventions, which we referred to as toolkits, to ameliorate excess emotions. Based on my experiences as a science educator who had visited Taiwan, Singapore and China on numerous occasions, I felt certain that we could learn how to ameliorate emotions, when and as necessary, by studying knowledge systems such as acupressure and reflexology. This became an activity that I began immediately and pursued intensively on visits to Taiwan and Singapore. I first learned about JSJ from a science educator in Singapore. She was helping me to learn about Chinese medicine, and particularly about the potential of reflexology to ameliorate intense emotions. I was learning about acupressure and reflexology by 
receiving treatments, seeing firsthand whether and how these knowledge systems were successful. My colleague told me about her experiences with JSJ and shared some handouts and The Touch of Healing (Burmeister and Monte 1997). In my studies of complementary medical knowledge systems, JSJ struck me as highly relevant to our interests in improving literate citizenry and wellness while deepening our understandings of emotions and mindfulness.

\section{Jin Shin Jyutsu}

As a knowledge system, JSJ can be traced back to India, before the birth of Buddha. Some of the ancient knowledge was preserved in Pali language and paintings, statues, and jewelry. In the early part of the 20th century a philosopher, Jiro Murai, from Japan, retrieved some of the JSJ knowledge and substantially enhanced it through empirical work with humans and other animals. Two of his students/apprentices, Mary Burmeister and Haruki Kato, learned from Murai, collaborated with him after an initial experience, and continued to develop ISJ with an expanding network of scholars after his death. Following the passing of Burmeister and Kato, the expansion and refinement of JSJ continued in both the US and Japan and its global dissemination as a complementary medical practice is ongoing.

We regard JSJ as a knowledge base that has been refined over thousands of years to promote wellness and ameliorate excess emotions (Tobin et al. 2015). The underlying theory relates to Qi, universal energy, and its flows through the body. In the case of humans there are 26 pairs of safety energy locks (SELs) through which Qi flows (Fig. 2), providing the life source to the body. JSJ identifies flows through the SELs to show movement of Qi through the body from top to bottom, side to side, and to each of 12 organs (e.g., heart, lung). When a body is disharmonized, energy can be blocked at or close to the SELs, thereby disrupting one or more of the flows needed to distribute the life force to different parts of the body. The experience for an individual when blockages occur might be abnormal functioning of organs, illness, and different parts of the body being susceptible to damage (e.g., fracture of a bone). Using the principles of JSJ, an individual can use selfhelp techniques or a JSJ practitioner can use particular holds to restore harmony to the body and get various flows moving smoothly. In the past few years we have studied human conduct with JSJ as one of the components of a multilogical framework (e.g., Tobin et al. 2015).

When I first began to learn JSJ I attended classes at the cancer center of the Morristown Medical Center in New Jersey, USA ${ }^{4}$. This was significant to me because the hospital offered JSJ to patients as supplementary treatments, especially in the post-operative phase. Furthermore, the hospital is collaborating with the JSJ headquarters in Arizona (USA) to offer a certificate program in JSJ - an indication of its growing acceptance by some parts of the Western Medicine system. At this New Jersey hospital there is tangible evidence of acceptance of an integrative approach to health - including Western Medicine and the option of treatments of complementary practices, including JSJ. As an example, when a friend of mine had a double mastectomy at the Morristown hospital I offered her JSJ as a way to afford a successful transition to good health. She smiled and let me know that the hospital offered her the same choice, which she accepted and was now receiving JSJ in a post-operative program. The Markey Cancer Center at the University of Kentucky (http://ukhealthcare.uky.edu/Markey/jsj/) offers a similar complementary approach to cancer treatment. 


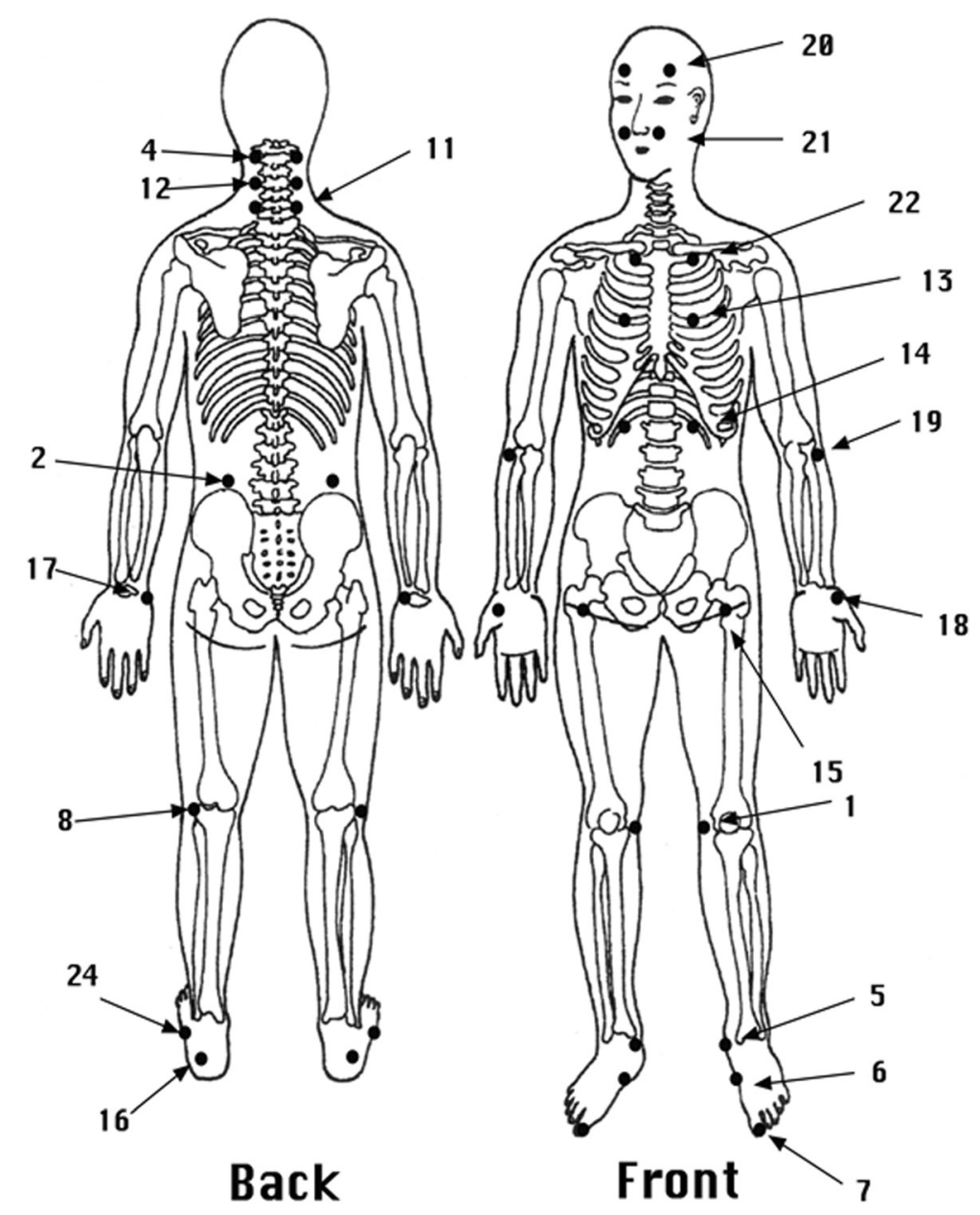

Fig. $\mathbf{2}$ Selected SELs that have salience to this research

In several studies we have shown that participants touch and hold parts of the body in places that correspond to SELs that can be used to ameliorate emotions, reduce high pulse rate, and maintain a state of calm and control (Tobin 2015). Also, we have used our knowledge of JSJ to develop interventions such as meditation and wellness toolkits. These JSJ-oriented interventions will be used in ongoing research. At the same time, we are using JSJ flows to address important wellness projects such as diabetes 2 . In this study I collaborate with a colleague, who has diabetes 2, to identify JSJ flows that reduce blood sugar by approximately $35 \%$. In addition, my colleague has reduced insulin dosage and other prescribed medications by $50 \%$. Obviously, the goal is to work with Western practitioners to reduce the impact diabetes 2 has on lifestyles.

There are many priorities for research, including problems of aging, back, shoulder and neck injuries, allergies, weight loss, knee and hip problems, and headaches. Our goal is to document how human wellness can be enhanced by the use of JSJ through self-help and JSJ practitioners. In laying out these ideas for research, I embrace a high value for educating the citizenry at large, ensuring that what is taught and learned is highly relevant to literate citizenry and well-being of the planet and the myriad life forms it supports. 
In our decision to include JSJ as part of a multilogical bricolage we are not taking a stance on what is true and assuming there is only one framework to promote human wellness. We accept that Western medicine has made amazing progress and has shown itself to be very effective in diagnosing and treating many human ailments. We do not offer JSJ as an alternative or as superior. Instead, we regard JSJ as a way to look at human wellness and address disharmonies as and when it is appropriate to do so. We do not regard it as useful to examine/critique JSJ through the lenses of Western medicine. Instead, the tools for reviewing and refining JSJ are within JSJ (Kaptchuk 2000). Ted Kaptchuk (2000) addresses the issue of critiquing a knowledge base such as Chinese medicine arguing that:

Chinese medicine is a coherent system of thought that does not require validation by the West as an intellectual construct. Intellectually, the way to approach Chinese concepts is to see whether they are internally logical and consistent, not to disguise them as Western concepts or dismiss them because they do not conform to Western notions (p. 77).

The acceptance of knowledge systems as viable is inextricably linked to power relationships and frequently involves politics. For example, Craig Janes (1995) addressed the legitimacy of Tibetan Medicine in ways that highlight the quandary faced by those who seek to employ JSJ and other knowledge systems considered to be viable as complementary with Western medicine. Janes noted:

At times Tibetan medicine has been repudiated in official revolutionary discourse as superstition employed by the corrupt feudal elite in its exploitation of peasants and nomads. At other times it has been championed as a valuable resource to the masses, a respected member of the family of "Chinese medicines." These radical shifts in revolutionary discourse have been accompanied by wide swings in the institutional legitimacy of Tibetan medicine. Tolerated but ignored from 1951 to 1962, Tibetan medicine was officially sanctioned as a component of the public health system in 1962 and given funds for clinical operations and training programs. Four years later, Tibetan medicine was again delegitimized, and by 1978 it was perched on the edge of institutional extinction. By 1985 Tibetan medicine emerged again as a legitimate sector of the government health bureaucracy and today plays a significant role in the provision of primary health care throughout the region with a substantial operating budget and over 1200 practicing physicians. (Janes 1995, p. 7).

As is evident in the struggles for legitimacy of Tibetan medicine, power relationships will be central to efforts to expand the agenda of science educators to include JSJ as a knowledge system that can contribute to the wellness and well-being of living things. Accordingly, there are risks for those who rise to the challenge of transforming science education by joining my colleagues and me in a quest to promote wellness and well-being of all by including JSJ and other complementary medical knowledge systems in multilogical research on wellness and well-being.

\section{Mindfulness and meditation}

Our work on mindfulness occurs at a volatile time in which there is an exponential increase in studies involving mindfulness and applications that have leapt ahead of research 
(Powietrzynska and Tobin 2015). So much is happening. In our own research and practice we have learned a great deal. A subtle aspect of what we have learned is that the multidimensionality of mindfulness has never been more apparent. We spent several years developing heuristics for use in research, and in so doing, have pushed to develop characteristics that can be represented verbally and others that transcend language, involving visual and auditory resources, such as art and music, for example.

Initially we endeavored to identify as many characteristics as we could and eventually we realized that, since they are constituents of a whole, efforts to enhance mindfulness need only focus on a relatively small number of characteristics. Because of the close relationship on emotion to wellness, my strong interest, which I will pursue, concerns not getting stuck on emotion, and, if an emotion sticks to conduct; how to get unstuck (Chödrön 2006).

Meditation is closely related to my interest in getting and staying unstuck. One of the high-priority foci I have for my research is to identify interventions to reharmonize the body when disharmonies have occurred, presumably due to an excess of emotions creating physiologic responses such as stress, elevated pulse rate, low blood oxygenation, high blood sugar composition, and elevated blood pressure.

My initial attempts at meditation seemed fraught. I did not have a meditation teacher and based my practice on imagistic focus - in my case on a red 3. The activity was not without a steep learning curve as I discovered other ways to focus, set aside wayward thoughts, and eventually how to release my mind from focus. Without effort I seemed able to enter a dreamlike state that was very passive and relaxing. My reading on meditation led me to breathing meditation and I adopt a practice of using mala beads to alert me to the end of one (108 breaths) or two (216 breaths) cycles of abdominal breaths, and thereby to allow me to maintain focus on the breath rather than on counting. I also learned from reading and personal experience in doing breathing meditation, about its benefits, especially relating to neuroplasticity and emotional styles (Davidson and Begley 2012). I was delighted about using meditation to enhance mindfulness, remove attachments, and stay in the moment. And, at a personal level, I found it convenient to use short periods of breathing meditation to ameliorate excess emotions and unattach emotions and conduct- especially to reduce frustration and stress.

We have described elsewhere how we used breathing meditation as an intervention to increase mindfulness in a study situated in a teacher education program at Brooklyn College, USA (Tobin et al. 2015). Based on some initial success we disseminated what we learned in the Brooklyn College study to the classes we were teaching - in my case doctoral classes at the Graduate Center, research meetings, and at our regular monthly research seminar. In each case I implemented a 5-min breathing meditation at the start of each activity. The goal was to initiate a higher state of mindfulness to enhance teaching and learning in these similar, but distinctive activities. Furthermore, the adoption of this approach was a sign to participants that dissemination from research to lifeworlds is highly desirable.

As we have learned more about the relationships between emotions and physiology we have used JSJ to restore harmony to unbalanced/asynchronous universal pulses. As the pulses synchronize, many aspects of the body can change. Accordingly, we have started to use ISJ holds as part of the 5-min meditation we use in our day-to-day 
practice as scholars. For example, we have used holds that are known to reduce tension, clear the mind, and assist breathing. These are promising practices waiting to be supported by research. However, since there is a vast empirical support for ISJ, we anticipate that the use of JSJ-orientated meditations would enhance mindfulness and well-being.

\section{Next steps}

Priorities for science education must change to address lifestyles and sustainability. In this paper I argue for forms of inquiry that are grounded in everyday life. To the extent that people's lives are uncertain the research we do in science education needs to be responsive to contingencies and attentive to the unfolding aspects of life. Foci for inquiry emerge from what people do in their lifeworlds, events that arise and are considered by participants to be noteworthy. The multilogical methodologies I advocate in this paper are flexible and can accommodate the many meaning systems used by Earth's citizens in their everyday lives.

Valuing difference as a resource for doing and learning from research is a necessary component of adopting collaborative models in which all participants do research for the purpose of improving the quality of life. Different theoretical frames are used to illuminate experience, identify events, and provide rationale for what participants consider to be happening and of value. Authentic inquiry serves as a heuristic for doing research that benefits all participants, respects difference, and seeks to remove disadvantage. Dissemination of what is learned from research considers participants and those with whom they interact in social life as central. With a focus on learning from others and educating all participants about what has been learned from research, authentic inquiry is both transformative and expansive, taking advantage of ripple effects as participants change practices as they interact with others in the world. In so doing they expand the possibilities for those with whom they interact to learn from the interactions and thereby benefit from the research.

In laying out my advocacy for a multilogical bricolage I presented authentic inquiry, event-oriented inquiry, emergence and contingence, multilectical relationships, and polysemia as critical components. Consistent with our ongoing research I used JSJ as a knowledge system that is not mainstream and can be used to expand possibilities for human wellness in ways that promote self help, assisting others to improve wellness, and increase knowledge and awareness of relationships between health and emotions - in a context of everyday life. Importantly, JSJ is not presented as an alternative to Western medicine but as a complementary way of seeing wellness in the world and expanding the possibilities for action in its many forms - including research. The components of a multilogical bricolage are not systems of truth, but are ways of seeing the world and making sense of what happens. By heightening awareness in new ways, possibilities for action are expanded and new directions are forged.

There is a lot involved in valuing difference as a resource for learning. As scholars we should take difference seriously. Examine the epistemological issues of what counts as knowledge, considering transcendence and crises of representation. This cannot be done in isolation of axiology since different facets of epistemology might be valued more highly than others. Such differences can make a big difference to what people do as they enact life. Also, polysemia is much more than a quaint object for inquiry. 
Others have their own ontologies that are reflected in how they experience the world and respond to questions like what is happening and why it is happening. As we embrace all participants as researchers there is a necessity for respect and compassion about all lives and the knowledge systems that support them.

From my standpoint all forms of enactment are cultural production, simultaneously transformative and reproductive. Accordingly, the next steps for science education will be both transformative and reproductive. Given the precarious state of humanity it behooves science educators to closely connect their scholarship to the challenges that face humanity - including sustainability, climate change, and wellness. There is a necessity for all science educators to act locally while they think globally. What are the priorities for research in science education in your local context? Before anyone answers this question, I suggest $\mathrm{s} /$ he examines the birth through death continuum and select participants from whom we can best learn and whose practices can change in an endeavor to enhance issues such as sustainability and wellness.

\section{Questions and answers}

Question: to what extent has your research methodology being influenced by Asian scholars and knowledge systems?

Many of the central tenets of our ongoing research on emotion and wellness are grounded in Asian knowledge systems and the work of colleagues in different Asian countries. For example, I met Chao-Ti Hsiung and Hsiao-Lin Tuan when they were doctoral students at the University of Georgia in the 1980s and undertook collaborative research with them at that time. In 1992 I made my first visit to Taiwan to work with science educators throughout the country on the use of interpretive research and science education. As well as presenting workshops in Taipei, Chunghua, and Kaohsiung, I interacted intensively with leading Taiwanese science educators, including Jong Hsiang Yang. It was during these myriad conversations that I experienced dialectical thought in everyday life experiences. For example, as Yang wisely pointed out, to successfully drive in Taipei, it is necessary for drivers to have both patience and courage. Too much patience and you never move; too much courage and you don't get far. To be successful you need both courage and patience, each having the right measure.

In successive trips to Taiwan over an interval of almost 20 years I presented the latest findings from my ongoing research and associated methodologies and methods. At the same time I was vigilant for learning opportunities, paying attention to lifestyles in everyday practices as well as what was happening in schools and research. I visited many temples on numerous trips to different Taiwanese cities and became fascinated by Buddhist philosophy and ways of describing life. I experienced reflective and contemplative aspects of Buddhism and over the years, and as their appeal grew stronger, my reading expanded to include meditation and mindfulness. In addition, I often made appointments to receive Chinese massage and acupuncture, thereby paving the way to better appreciate what could be accomplished with these practices and other Eastern knowledge systems that related to health and wellness.

Chia-Ju Liu, from Taiwan's National Kaohsiung Normal University, is a leader in making connections between science education and the medical sciences. Her research introduced me to social neuroscience as a discipline and opened the door for me to develop ideas of multilevel analysis. One of her early studies, which focused on physics education as a 
hedge against the onset of Alzheimer's disease (Liu 2010), was a catalyst for our work that focused on science education across the birth through death continuum.

While in Singapore in 2012, Poh Hiang Tan introduced me to the work of Ajahn Brahm (2006), a Buddhist monk who was ordained in the Northeast of Thailand. Coincidentally, he had built a monastery's just outside of Perth in Western Australia. Also, since he had been a physics teacher in the United Kingdom, before becoming a monk, I was interested in his biography and his writing about meditation. His writing expanded ideas we were pursuing on uses of breathing meditation as a way to expand mindfulness in education. Prior to our becoming aware of Brahm's work our knowledge about meditation was grounded in yoga (McGonigal 2009) and Tibetan Buddhism (Chödrön 2006). We were well aware of the connection between breathing meditation, neuroplasticity, and health because of the work that Richard Davidson (2010) and his colleagues were doing with the Dalai Lama. These collaborative studies were beginning to break down barriers between science and religion. Brahm's work pushed us to think about alternative ways of meditating and different outcomes or states of being. The implications for mindfulness and our associated research assisted us to integrate meditation, mindfulness and wellness is our ongoing research.

Although I address our studies of JSJ earlier in the paper, I emphasize here that I first learned about the knowledge system from Poh Hiang Tan during my 2012 visit to Singapore. Subsequently I was educated about JSJ in the United States, but hasten to add that the retrieval and expansion of JSJ was initiated in Japan in the 20th century.

Clearly I have learned a great deal from scholars in Asia and experiences from numerous visits to Asian countries - most recently Thailand. It is important that science educators are open to opportunities to learn from people in Asia, including many fine scholars. Although the primary purpose of a visit might be to serve as consultant, keynote speaker, etc., a multilectical relationship that includes "| learner" is always in play. What is necessary, is to set aside the identity element of expert and emphasize as co-equal the idea of learning from others by being "in-with-others".

\section{Question: should science educators maintain sharp divisions between science and religion?}

As I emphasized in the paper, we regard theoretical frameworks as complementary to one another - affordances for looking, seeing, experiencing, and making sense. We do not regard them as truth systems and acknowledge that they not only illuminate aspects of social life, but they also obscure. The problem does not arise if there is acceptance of polysemia and high value is assigned to learning from difference.

I believe that there is a great deal to be learned from what I consider to be "lost knowledge systems." For example, an important component of JSJ is the use of mudras (finger holds) to restore disharmonies associated with illness (Hirschi 2000). It is possible that the knowledge is contained in art work - including paintings and other artistic objects such as statues and decorative paintings, murals, and jewelry. I have spent hours in temples and monasteries in different Asian countries examining the orientations, touches and holds of arms, hands, fingers, legs, feet, and toes. As fresh insights emerge I consider what I am learning in relation to ongoing research on emotion and wellness.

Our examples of meditation, mindfulness, and the JSJ concern knowledge systems that originated in Asia and were developed by people affiliated with religion - including Buddhism and Hinduism. These knowledge systems are useful as part of a bricolage and 
are considered as complementary to mainstream knowledge systems - not as unitary truth systems or master narratives.

\section{Question: what are the risks for junior faculty to follow your suggestions to adopt a multilogical methodology?}

A key issue is isolation - separation from a community. Another way to say this is to emphasize the importance of all scholars connecting with, creating, and expanding scholarly communities in the Academy. It is essential for junior scholars to be able to demonstrate that their work is peer-reviewed and has impact. In a context of science education the creation of new journals is in large part a response to dissatisfaction with a mainstream set of journals that were virtually indistinguishable. Risks within the Academy are usually associated with being unable to publish in peer-reviewed journals and an inability to establish a record of impact through citation and acknowledgment. Accordingly, junior scholars should work with their mentors to become well connected in networks and benefit from the peer support that goes with belonging to a scholarly community.

Within an institution it is wise for junior scholars to know their colleagues, especially those who will advise and vote on promotion, tenure, and other career milestones. Long before decisions are to be made, it behooves junior scholars to know about colleagues' scholarship, including where they get their funding, what they do, what methodologies frame their work, and where they publish. Reading their published material and having conversations with them about it also seems wise. Furthermore, in this electronic age, it makes sense to know your colleagues in terms of their citation record, h-index, and standing in communities such as Google Scholar, Research Gate, and academia.edu. Personally I use the software Publish or Perish (Harzing 2007) to view data associated with a relatively large pool of colleagues and former students. I do not do this with malicious intent. It is just that it helps me to know the professional standing of colleagues Similarly, it will likely assist junior scholars to know about colleagues who offer advice and vote on committees that make a difference to their careers.

So, to sum up, there are risks. Senior colleagues will advise and judge based on their value systems. Accordingly, well before advice is given and official judgments are rendered, it is time to initiate dialogues so that junior scholars can lay out their research and its associated foundations and possibilities. Junior scholars need to establish spaces at the local level to educate colleagues about them, show their work is credible, and if necessary, demonstrate the viability of their scholarship in a mainstream flux of angry hornets!

\section{Endnotes}

${ }^{1}$ http://www.gutenberg.org/files/23772/23772-h/23772-h.htm\#Page_108

${ }^{2}$ The vertical bar $(\mid)$ is used in our research program to show a dialectical relationship between two constructs, in this case agency and structure, that are assumed to be constituents of a whole, recursively related to one another, and coexist - each presupposing the other.

${ }^{3}$ I use we to describe frameworks used in the research in which I have engaged. We is an appropriate acknowledgement of the collective aspects of social life in which all knowing is social distributed. 


\section{${ }^{4}$ http://www.atlantichealth.org/simon/our+services/additional+resources/support+ser vices/integrative+medicine+programs}

\section{About the Author}

Kenneth Tobin came to the Urban Education doctoral program at the Graduate Center of CUNY in the fall semester of 2003. Prior to that he had positions as tenured full professor at Florida State University (1987 to 1997) and the University of Pennsylvania (1997 to 2003). Also, he held university appointments at the Western Australian Institute of Technology (now Curtin University), Mount Lawley College and Graylands College (now Edith Cowan University). Before he became a university science educator in Australia in 1974, Tobin taught high school physics, chemistry, biology general science, and mathematics for 10 years. He began a program of research in 1973 that continues to the present day - teaching and learning of science and learning to teach science.

\section{Received: 23 September 2015 Accepted: 1 October 2015}

Published online: 21 December 2015

\section{References}

Bakhtin, M. M. (1986). Speech genres \& other late essays (Translated by V. W. McGee. Edited by C. Emerson \& M, Holquist). Austin: University of Texas Press.

Bellocchi, A., Ritchie, S. M., Tobin, K., Hudson, P., King, D., Sandhu, M., et al. (2014). Emotional climate and high quality learning experiences in science teacher education. J Res Sci Teach, 51(10), 1301-1325. doi:10.1002/tea.21170.

Bourdieu, P. (1992). The practice of reflexive sociology (The Paris workshop). In P. Bourdieu \& L. J. D. Wacquant (Eds.), An invitation to reflexive sociology (pp. 216-260). Chicago, IL: The University of Chicago Press.

Brahm, A. (2006). Mindfulness, bliss, and beyond: A meditator's handbook. New York: Wisdom Publications, IMC.

Burmeister, A., \& Monte, T. (1997). The touch of healing: energizing body, mind, and spirit with the art of Jin Shin Jyutsu* New York: Bantam Books.

Ceballos, G., Ehrlich, P. R., Barnosky, A. D., García, A., Pringle, R. M., \& Palmer, T. M. (2015). Accelerated modern humaninduced species losses: entering the sixth mass extinction. Sci Adv, 1, e1400253. 19 June 2015.

Chödrön, P. (2006). Getting unstuck: Breaking your habitual patterns \& encountering naked reality (audio CD). Louisville, CO: Sounds True.

Collins, R. (2004). Interaction ritual chains. NJ: Princeton University Press.

Davidson, R.J. (2010). Empirical explorations of mindfulness: conceptual and methodological conundrums. Emotion, 10(1), 8-11.

Davidson, R.J., \& Begley, S. (2012). The emotional life of your brain. London: Hodder \& Stoughton.

Erickson, F. (1986). Qualitative research on teaching. In M. C. Wittrock (Ed.), Handbook of Research on Teaching (3rd ed., pp. 119-161). New York: Macmillan.

Fellner, G. (2014). Broadening our lenses of perception to advance learning: An introduction to multilectics. Teach Teach Educ, 37, 169-182. doi:10.1016/j.tate.2013.04.015.

Garfinkel, H. (1967). Studies in ethnomethodology. Englewood Cliffs, NJ: Prentice Hall.

Geertz, C. (1973). The interpretation of cultures. New York: Basic Books.

Guba, E. G., \& Lincoln, Y. (1989). Fourth generation evaluation. Thousand Oaks, CA: Sage Publications.

Harzing, A. W. (2007). Publish or perish. Available from http://www.harzing.com/pop.htm. Accessed date 20 September 2015.

Hirschi, G. (2000). Mudras: Yoga in your hands. San Francisco: Weiser Books.

Hurd, P. D. (1997). Inventing science education for the new millennium. New York: Teachers College Press.

Janes, C.R. (1995). The transformations of Tibetan medicine. Med Anthropol Q, 9(1), 6-39.

Kaptchuk, T. J. (2000). Chinese medicine: The web that has no weaver. London: Rider.

Kincheloe, J.L., \& Tobin, K. (2009). The much exaggerated death of positivism. Cult Stud Sci Educ, 4, 513-528. doi:10.1007/s11422-009-9178-5.

Kuhn, T. S. (1996). The structure of scientific revolutions (3rd ed.). Chicago: The University of Chicago Press.

Liu, C. J. (2010). Alzheimer's disease. Unpublished manuscript, National Kaohsiung Normal University.

Matthews, M. R. (Ed.). (1998). Constructivism in science education. Dordrecht, Netherlands: Kluwer Academic Publishers.

McGonigal, K. (2009). Yoga for pain relief: Simple practices to calm your mind and heal your chronic pain. Oakland, CA: New Harbinger Publications.

Mueller, M., \& Tippins, D. J. (2015). Ecojustice, citizen science and youth activism. Dordrecht: Springer.

Phillips, D.C. (1983). After the wake: postpositivistic educational thought. Educ Res, 12(5), 4-12.

Piaget, J. (1964). Development and learning. J Res Sci Teach, 2, 176-186. http://dx.doi.org/10.1002/tea.3660020306. Accessed date 20 September 2015

Powietrzynska, M., \& Tobin, K. (2015). Mindfulness and science education. In R. Gunstone (Ed.), Encyclopedia of science education (pp. 642-647). Dordrecht: Springer. doi:10.1007/978-94-007-6165-0_264-2.

Powietrzynska, M., Tobin, K., \& Alexakos, K. (2015). Facing the grand challenges through heuristics and mindfulness. Cult Stud Sci Educ, 10, 65-81. doi:10.1007/s11422-014-9588-x.

Roth, W.-M., \& Tobin, K. (2010). Solidarity and conflict: prosody as a transactional resource in intra- and intercultural communication involving power differences. Cult Stud Sci Educ, 5, 807-847. doi:10.1007/s11422-009-9203-8.

Sewell, W.H., Jr. (1992). A theory of structure: duality, agency and transformation. Am J Sociol, 98, 1-29.

Sewell, W.H., Jr. (1997). Geertz, cultural systems, and history: from synchrony to transformation. Representations, 59, 35-55.

Sewell, W. H., Jr. (1999). The concept(s) of culture. In V. E. Bonell \& L. Hunt (Eds.), Beyond the cultural turn (pp. 35-61). Berkeley, CA: University of California Press.

Sewell, W. H., Jr. (2005). Logics of history: Social theory and social transformation. Chicago: University of Chicago Press. Swidler, A. (1986). Culture in action: symbols and strategies. Am Sociol Rev, 51, 273-286.

Tobin, K. (1986). Effects of teacher wait time on discourse characteristics in mathematics and language arts classes. Am Educ Res J, 23(2), 191-200.

Tobin, K. (2000). Becoming an urban science educator. Res Sci Educ, 30, 89-106. 
Tobin, K.G. (2008). In search of new lights: getting the most from competing perspectives. Cult Stud Sci Educ, 3, 227-230. Tobin, K. (2015). The sociocultural turn: Beyond theoretical imperialism and the imperative of learning from difference. In C. Milne, K. Tobin, \& D. deGennaro (Eds.), Sociocultural studies and implications for science education (pp. 3-31). Dordrecht, The Netherlands: Springer. doi:10.1007/978-94-007-4240-6_1.

Tobin, K., \& Alexakos, K. (2013). The cogenerative dialogue heuristic. NY: The Graduate Center of the City University of New York.

Tobin, K., \& Capie, W. (1982). Relationships between classroom process variables and middle school science achievement. J Educ Psychol, 74, 441-454.

Tobin, K., \& Llena, R. (2011). Producing and maintaining culturally adaptive teaching and learning of science in urban schools. In C. Murphy \& K. Scantlebury (Eds.), Coteaching in international contexts: Research and practice (pp. 79-104). Dordrecht: Springer.

Tobin, K., \& Ritchie, S.M. (2011). Multi-method, multi-theoretical, multi-level research in the learning sciences. Asia Pac Educ Res, 20(3), 117-129.

Tobin, K., \& Roth, W.-M. (2006). Teaching to learn: A view from the field. Rotterdam, NL: Sense Publishing.

Tobin, K., Roth, W.-M., \& Zimmermann, A. (2001). Learning to teach in urban schools. J Res Sci Teach, 38, 941-964

Tobin, K., Alexakos, K., \& Powietrzynska, M. (2015). Mindfulness and wellness: central components of a science of learning. Innovación Educativa, 15(67), 61-87.

Turner, J. H. (2002). Face to face: toward a sociological theony of interpersonal behavior. Palo Alto: Stanford University Press.

Submit your manuscript to a SpringerOpen ${ }^{\circ}$ journal and benefit from:

- Convenient online submission

- Rigorous peer review

- Immediate publication on acceptance

- Open access: articles freely available online

- High visibility within the field

Retaining the copyright to your article

Submit your next manuscript at $>$ springeropen.com 